\title{
Effect of Integrated Medicine on Physical Performances of Orthopaedic and Stroke Patients: A Propensity Score-Matched Study
}

Simonetta Bernardini ${ }^{1^{\star}}$, Anna Gottard ${ }^{2}$, Massimo Rinaldi ${ }^{1}$, Alessandra Mattei ${ }^{2}$, Gianni Virgili ${ }^{3}$, Franco Cracolici ${ }^{1}$, Rosaria Ferreri ${ }^{1}$ and Roberto Pulcri ${ }^{1}$

${ }^{1}$ Department of Integrated Medicine, Centre of Integrated Medicine Pitigliano Hospital, Italy

${ }^{2}$ Department of Statistics, Computer Science Applications, University of Florence, Italy

${ }^{3}$ Department of Translational Surgery and Medicine, University of Florence, Italy

Corresponding author: Simonetta Bernardini, Centre of Integrated Medicine Pitigliano Hospital, Via N. Ciacci, 340. 58017, Pitigliano, Grosseto, Tel: 00393407291939; Fax: 39055280747; E-mail: bernardini@s-bernardini.it

Rec date: 05 November 2017, Acc date: 14 November 2017, Pub date: 20 November 2017

Copyright: (c) 2017 Bernardini S, et al. This is an open-access article distributed under the terms of the Creative Commons Attribution License, which permits unrestricted use, distribution and reproduction in any medium, provided the original author and source are credited.

\begin{abstract}
Background: To evaluate the clinical efficacy of treatment with integrated medicine (acupuncture and homeopathy in addition to conventional treatment) in improving measures of physical ability. Data from medical records with patient-reported information on background characteristics, diagnosis on admission (orthopaedic conditions and stroke) and movement performance indices.

Methods: Comparative, observational, propensity-score matched clinical study. Participants: 383 adults admitted to the rehabilitation Centre in Manciano, stratified by stroke and orthopaedic condition. Interventions: acupuncture and homeopathy with physical rehabilitation vs. rehabilitation alone. Main outcome measures: Activities of daily living performance indices for patients with knee or hip replacements (Barthel index) and stroke patients (Barthel index and Trunk control test). Number of days of analgesic drug treatment for orthopedic patients.
\end{abstract}

Results: The activities of daily living performance indices showed a significant improvement for both stroke patients (Fisher p-value: 0.008 and 0.046 for Barthel index and Trunk Control Test respectively) and orthopaedic patients (Fisher $p$-value: 0.032 for Barthel index) treated with integrated medicine. The treatment also reduced the duration of analgesic treatment by 2.8 days (Fisher p-value: 0.0015 ).

Conclusion: Integrated medicine helps to improve rehabilitation performance in stroke and orthopaedic patients and considerably reduces the need for analgesic drugs.

Keywords: Acupuncture; Homeopathy; Integrated medicine; Stroke rehabilitation; Orthopaedic rehabilitation

\section{Introduction}

In February 2011 the first Italian Hospital Centre of Integrated Medicine (IM) was opened in Pitigliano Hospital and in the nearby neurological and orthopaedic rehabilitation centre in Manciano, Tuscany (Italy). The main purpose of the project was the close cooperation of conventional doctors, doctors specialised in homeopathy and acupuncture and healthcare personnel under an IM treatment model [1]. The project was financed by the Tuscany regional health authority with the aim of verifying the efficacy of complementary and alternative medicine (CAM) in healthcare.

To our knowledge the clinical setting we adopted (orthodox medicine plus homeopathy and acupuncture) is totally new. It is in fact completely different from other apparently similar settings reported in the literature, such as that used by Fang et al. [2], who report on a protocol for gathering data from the simultaneous use of acupuncture and phytotherapy. The main novelty of the present project is that it was implemented within the public health system in rehabilitation, neurological and orthopaedic centre.
We report on the data collected from patients admitted to this centre after knee or hip replacement (orthopaedic patients) or after a stroke (stroke patients) that, in addition to standard medical treatment, also received homeopathic and acupuncture therapies. The patients' rehabilitation performance and activities of daily living performance indices were assessed (as specified below) and statistically evaluated using a potential outcome approach to causal inference.

\section{Methods}

\section{Design and data source}

The data analysed herein concern patients admitted to the rehabilitation centre in Manciano (Italy) between 2010 and 2012. Data for later years is not used because the main activity performance index was replaced in mid-2012 by the hospital administration through a decision not related to this study. Data are derived from inpatient records filled out by hospital staff in line with hospital guidelines.

Inpatients admitted in 2010 received only the ordinary therapy (OT) for rehabilitation, and act as control patients. Some of the inpatients admitted in 2011-2012 also received the IM treatment (homeopathy and acupuncture), and are hereinafter indicated as the treated patients. 
For organisational reasons the IM treatment was proposed only to patients admitted on specific days of the week, Tuesday, Wednesday and Thursday, when the team of IM doctors was present in the centre. Patients admitted in 2011-2012 who were not allocated to IM treatment were not included in the study, as they could have had access to the homeopathic therapy, possibly invalidating the analysis.

The relevant patients received information about the IM treatment and signed an informed consent form if they agreed to it. $98 \%$ of the inpatients accepted the IM treatment.

\section{IM protocols}

\section{Homeopathic protocol}

We chose a "tailored therapy" using a magistral formulation adapted for neurological sequelae and orthopaedic surgery. For homeopathic magistral preparations, the active substance was chosen on the basis of homeopathic similarity to actual symptoms, the "reactive model" of the disease and the "constitution" of the patient. The decision to use a magistral formulation was based on its practical advantages: better patient compliance, especially in the case of elderly patients and those not accustomed to taking homeopathic medicines; reproducibility of the treatment protocol, and reduced cost (about seven euros per patient per week).

\section{Acupuncture protocol}

Traditional and microsystem acupuncture were used. The combination of three techniques (traditional acupuncture, Yamamoto, and sujok) offers crucial support in neurology and orthopaedics, due to the flooding of a cascade of neurotransmitters (beta endorphins, serotonin, etc.), thus rivalling conventional therapies in terms of efficacy.

Both the OT and the IM treatments were carried out regularly during the observational period and were administered following a personalised rehabilitation protocol established by the clinic guidelines. The IM patients received two acupuncture sessions a week and homeopathic medicines daily, as prescribed by the homeopathic doctors and prepared by the hospital pharmacy's galenics department. All IM patients were also treated with the routine OT therapy in use at the rehabilitation centre.

The patients were divided into two groups: the orthopaedic group included patients with knee or hip replacements, and the stroke group included patients who had suffered ischaemic or haemorrhagic strokes. Both IM and control patients stayed in the centre for about three weeks (stroke group) or two weeks (orthopaedic group), in line with the rehabilitation centre's timescale.

\section{Variables}

Information on personal characteristics, such as gender and age, and clinical characteristics, including measures of physical ability, were collected from the inpatient records. The latter were measured upon both admission and discharge. The primary measure of interest was the Barthel index of physical disability [3]. The Barthel index gives scores between 0 and 20, with 20 signifying maximum ability. In addition, the trunk control test was administered to stroke patients, another measure of functional ability for these patients [4].

This test contains 12 four-point (from 0 to 3 ) items assessing difficulty in maintaining or moving between lying, sitting, and standing positions. The number of days of analgesic administration during hospitalisation was also recorded. All measures were taken by the clinic's personnel. The team of IM doctors was not involved in the evaluation process in any way.

\section{Statistical analysis}

The causal effects of IM treatment on activities of daily living performance indices were evaluated using a potential outcome approach to causal inference [5]. A causal effect of the treatment on each patient is defined as a comparison of potential outcomes under the IM treatment and the OT treatment, for each outcome variable. The fundamental problem of causal inference [6] is that at most only one of the potential outcomes can be observed for each patient, depending on the treatment actually received.

In this study, the outcome variables under the OT treatment could be observed for patients who were admitted in 2010, and under the IM treatment for those admitted in 2011-2012. Consequently, the treatment allocation mechanism was linked only to the date of admission, i.e. to the occurrence of the stroke or orthopaedic event. These circumstances are referred to as natural and it is reasonable to assume that patients were naturally randomly assigned to either the IM treatment or to the OT treatment group.

In any case, the effectiveness of this natural randomisation was checked with further analyses. To check the balance of covariates between the treatment groups, two approaches were applied: (1) calculation of the standardised differences for marginal comparisons and (2) calculation of a likelihood ratio (LR) test for a joint comparison.

The LR test compares a logistic regression model for the treatment that includes all covariates against a restricted logistic regression model that sets the coefficients of the covariates to zero.

Whenever at least one of these two comparisons failed, it was assumed that the sample came from a stratified randomised experiment, which is defined by a strong ignorable treatment assignment mechanism [7]. Strong ignorability means assuming that the treatment is randomly assigned within cells defined by the values of pre-treatment variables, which is a reasonable assumption in our study.

Under this assumption, randomisation inference for finite samples was used, computing Fisher exact p-values for sharp null hypotheses [8], which assume no effect of the treatment for any patients. As the aim was to establish if the IM treatment has beneficial effects, the alternative hypotheses were chosen as one-tailed: at least one patient exists whose potential outcome difference is strictly positive. These tests were conducted using the difference in average gain scores by treatment status as the test statistic.

The test statistics were formally defined as follows: the outcomes were first transformed into gain scores, defined as the difference between potential outcomes and the baseline measurement of the outcome. For instance, gain scores for the Barthel Index were defined as the difference between potential outcomes for the Barthel Index on the patient's discharge and the measurement of the Barthel Index on their admission.

The differences in the means of the transformed outcomes between treated and a control patient was then calculated. Fisher exact p-values were calculated using 20,000 randomly chosen permutations of the treatment allocation. All statistical analyses were computed using $\mathrm{R}$ Foundation software [9]. 
Citation: Bernardini S, Gottard A, Rinaldi M, Mattei A, Virgili G, et al. (2017) Effect of Integrated Medicine on Physical Performances of Orthopaedic and Stroke Patients: A Propensity Score-Matched Study. Altern Integr Med 6: 255. doi:10.4172/2327-5162.1000255

Page 3 of 6

\section{Results}

Tables 1 and 2 respectively summarize the statistics (means for continuous variables and proportions for binary variables) for stroke patients and for orthopaedic patients grouped by treatment status. Table 3 presents the LR test results.

In stroke patients (Table 1), pre-treatment variables are wellbalanced in the two treatment sub-groups: the sample means are similar, all the standardized differences are very small and the p-value for the LR test (Table 3) strongly indicates no evidence of influence of the pre-treatment variables on treatment allocation. In contrast, for the orthopaedic patients (Table 2), there are some differences in the pretreatment background variables between the IM group and the OT group. The standardised differences in the entire sample (denoted as "before matching" in Table 2) are quite large, especially for the Barthel index on admission, for which the standardised difference was 0.66. Moreover the p-value for the LR test (Table 3) shows strong evidence that the covariates are not well-balanced between the treatment groups. These results could be due to the small sample size, and it was decided to make an adjustment for the systematic differences in the background characteristics of orthopaedic patients via a matching procedure.

\begin{tabular}{|l|l|l|l|l|l|}
\hline \multicolumn{2}{|l|}{} & $\begin{array}{l}\text { Treated } \\
\mathbf{n = 4 6}\end{array}$ & $\begin{array}{l}\text { Controls } \\
\mathbf{n = 1 1 2}\end{array}$ & $\begin{array}{l}\text { Standardised } \\
\text { difference }\end{array}$ & Fisher P-value \\
\hline Pre-treatment variables & 70.13 & 70.91 & -0.08 & 0.657 \\
\hline Mean age & 0.72 & 0.66 & 0.12 & 0.579 \\
\hline Male to female ratio & 0.83 & 0.77 & 0.15 & 0.528 \\
\hline Ischaemic to haemorrhagic stroke ratio & 10.57 & 9.02 & 0.27 & 0.137 \\
\hline Barthel index (admission) mean & 61.15 & 57.03 & 0.14 & 0.443 \\
\hline Trunk control test (admission) mean & & & \\
\hline Outcomes & 15.00 & 11.98 & & \\
\hline Barthel index (discharge) mean & 76.85 & 68.05 & & \\
\hline Trunk control test (discharge) mean & & \\
\hline
\end{tabular}

Table 1: Stroke patients: comparison of variables with and without IM.

Under strong ignorability a subsample of orthopaedic patients with well-balanced covariates between the treatment groups was created using propensity score-matching methods. Propensity scores were computed by logistic regression including all the pre-treatment variables. Matching was based on the nearest neighbour procedure without replacement, using the matchit package [10]. The sample thus obtained consisted of 29 IM patients and 87 OT patients, considered as coming from a stratified randomised experiment with 29 blocks. Each block included one treated patient and three controls. The last two columns in Table 2 respectively show summary statistics for the matched controls and standardised differences after matching. The last line in Table 3 presents the LR test $p$-value for the matched sample. It can be seen that matching strongly improves the balance of pretreatment variables between the treatment groups.

\begin{tabular}{|l|l|l|l|l|l|l|}
\hline & $\begin{array}{l}\text { Treated } \\
\mathbf{n = 2 9}\end{array}$ & $\begin{array}{l}\text { Controls } \\
\mathbf{n = 1 9 6}\end{array}$ & $\begin{array}{l}\text { Standardised } \\
\text { difference } \\
\text { before matching }\end{array}$ & $\begin{array}{l}\text { Matched Controls } \\
\mathbf{n = 8 7}\end{array}$ & $\begin{array}{l}\text { Standardised } \\
\text { difference } \\
\text { after matching }\end{array}$ \\
\hline Pre-treatment variables & \multicolumn{5}{|l|}{} \\
\hline Mean age & 70.28 & 71.95 & -0.21 & 71.39 & -0.14 \\
\hline Male to female ratio & 0.34 & 0.33 & -0.06 & 0.33 & -0.16 \\
\hline Knee to hip replacement ratio & 0.52 & 0.53 & -0.02 & 0.52 & 0.02 \\
\hline Barthel index (admission) mean & 12.76 & 14.96 & -0.66 & 13.29 & 0 \\
\hline Outcomes & \multicolumn{2}{|l|}{} \\
\hline Barthel index (discharge) mean & 18.21 & 18.08 & & 17.7 & \\
\hline
\end{tabular}

Table 2: Orthopaedic patients: comparison of variables with and without integrated treatment. 


\begin{tabular}{|l|l|l|l|l|}
\hline Group & $\begin{array}{l}\text { LR test } \\
\text { Statistic }\end{array}$ & $\begin{array}{l}\text { Degrees of } \\
\text { Freedom }\end{array}$ & $\begin{array}{l}\text { Asymptotic } \\
\text { p-value }\end{array}$ & $\begin{array}{l}\text { Fisher's } \\
\text { p-value }\end{array}$ \\
\hline Stroke patients & 7.9467 & 7 & 0.3373 & 0.3692 \\
\hline Orthopaedic patients: entire sample & 15.751 & 4 & 0.0034 & 0.0037 \\
\hline Orthopaedic patients: matched sample & 1.7629 & 4 & 0.7793 & 0.7938 \\
\hline
\end{tabular}

Table 3: Balance check via LR test and Fisher's exact p-values for the sharp null hypothesis of balance between groups.

Table 4 presents the observed test values and the corresponding Fisher exact p-values for stroke patients and orthopaedic patients. All the observed values are positive, indicating that IM treatment increases activities of daily living performance indices in both stroke patients and orthopaedic patients, with p-values from Fisher's exact tests for stroke patients of 0.008 for the Barthel Index and 0.046 for the Trunk Control Test.

For orthopaedic patients, the p-value for the Barthel Index was 0.032. This indicates that the IM treatment has some effect on the Barthel Index, although the evidence against the null hypothesis is not very strong, probably due to the small sample size.

\begin{tabular}{|l|l|l|}
\hline Variables & $\begin{array}{l}\text { Mean } \\
\text { Difference }\end{array}$ & $\begin{array}{l}\text { Fisher's one-sided } \\
\text { p-value }\end{array}$ \\
\hline Stroke patients & & \\
\hline Barthel index & 2.91 & 0.008 \\
\hline Trunk control test & 1.47 & 0.046 \\
\hline Orthopaedic patients & & \\
\hline Barthel index & 1.03 & 0.032 \\
\hline
\end{tabular}

Table 4: Observed values of the mean difference in gain scores between treated and control patients and Fisher's exact one-sided p-values for the sharp null hypothesis of no treatment effect.

For orthopaedic patients, it was also investigated whether the IM treatment had an effect on the use and duration of analgesic therapy. In the OT group $73.6 \%$ of patients took analgesics, versus $58 \%$ in the IM group. On average, the IM patients took analgesics for 2.89 days less than the OT group. This difference is significant, with a Fisher onesided p-value in the matched sample of 0.0015 .

\section{Discussion}

The data from this study show that integrated medicine is a promising approach for the rehabilitation protocol of stroke patients and those who have undergone knee or hip replacement. Even though this approach requires further support from clinical data, analysis of the results suggests that IM enhances neurological and orthopaedic rehabilitation as well as reducing the need for analgesics following hip and knee replacement.

To our knowledge, there are no literature reports describing an analogous use of integrated medicine (homeopathy and acupuncture) alongside conventional medicine in a clinical setting. In fact, the main novelty of the present project is that it was implemented within the public health system in rehabilitation, neurological and orthopaedic centre.

Fang et al. [2] recently described a protocol to gather data on the simultaneous use of acupuncture and phytotherapy. The perceptions and attitudes of conventional rehabilitation doctors towards the use of complementary and alternative medicine (CAM) have also been investigated $[11,12]$. As there is an ever-increasing patient demand for CAM therapies, it is necessary for doctors to be informed about their use. For example, in Switzerland, patients with spinal cord injury have both a high demand for and high satisfaction with IM, especially homeopathy and acupuncture [13]. In the present project, CAM therapies are available to inpatients through funding provided by the Tuscany regional health authority for the purposes of both healthcare and scientific research.

There are very few published articles concerning the use of homeopathy in rehabilitation, and unfortunately they are inconclusive, with some demonstrating efficacy [14,15] and others inefficacy [16]. In relation to stroke rehabilitation, most studies involve acupuncture. Although a Cochrane study [17] concluded that there was inadequate evidence to draw any conclusions as to the efficacy of this methodology, a meta-analysis [18] demonstrates its efficacy and safety. Few studies integrating acupuncture into post-stroke rehabilitation therapy have been performed in western countries [19,20]: to date, most have been conducted in the East, where acupuncture is a common and acknowledged therapy [21-24].

To our knowledge there are no studies involving the use of both homeopathy and acupuncture in rehabilitation after hip or knee replacement; several studies show the efficacy of acupuncture alone [25-31], although here too the studies were carried out in the East. Moreover, a study carried out in Korea [32] describes the patients' difficulty in moving between two hospitals, one for conventional western therapies and one for CAM. That study also demonstrates that there are no conventional hospitals incorporating IM clinical settings in the East, either.

Overall, there are very few reports [33] of the use of homeopathy in rehabilitation after joint replacement. This makes our experience almost unique. We consider it very important that integrated therapy reduced the need for analgesic use in hip and knee replacement patients. In fact, i) only $58 \%$ of such patients required analgesics, compared with $73 \%$ in the group treated with conventional medicine alone; and ii) IM therapy reduced the duration of the analgesic treatment (mean reduction of about 3 days).

It must be stressed that in the present study homeopathy and acupuncture were always used together, as was indeed envisaged in the healthcare integration model decided upon by the regional health government. This precludes evaluation of the individual efficacy of each of these therapies. However, it gives us the opportunity to discuss 
the efficacy of an IM protocol, which is the purpose of the Pitigliano experimental healthcare project. It is interesting to note the high acceptance of IM ( $98 \%$ of those to whom the IM protocol was offered) by the population treated in the Manciano rehabilitation centre. This is a very small town, and it could easily be assumed that its residents had very little knowledge of IM. However, the small size of the hospital is an advantage for this kind of trial, as it permits close contact among inpatients, and between inpatients and medical staff. This closeness helps to spread awareness of the IM patients' improved rehabilitation performance. Given the clinical setting, harmony between the conventional medical personnel and the IM doctors was essential to the success of the project. This lack of conflict helped the inpatients trust integrated therapies.

In this paper, we compared the improvement of stroke and orthopaedic patients before and after the implementation of IM using validated scales. The improvement in rehabilitation performance was evaluated among patients affected by the same condition and with the same inclusion criteria. The results have been and continue to be very encouraging, and the cost of the integrated treatment has been very modest.

\section{Conclusions}

The small number of patients in this study does not permit definitive conclusions to be drawn with regard to the efficacy of IM in a neurological and orthopaedic rehabilitation centre. However, these preliminary results allow us to affirm that IM can be incorporated alongside conventional treatments in a public rehabilitation hospital. According to our results, based on causal inference, IM improves rehabilitation performance in inpatients recovering from stroke and joint replacement surgery and also reduces the need for analgesics. The expenditure for the purchase of homeopathic medicines and acupuncture stations was very modest.

\section{Acknowledgments}

The project was funded by the Tuscany regional health authority specifically for the purpose of healthcare and research to verify the efficacy of CAM. The authors would like to thank the administrative department at Grosseto Local Health Authority ASL 9, the former Head Physicians at the Manciano rehabilitation centre, Dr. N. Zei and Dr. A. Barbetti, and the healthcare professionals at the Manciano centre, for their ongoing support and help in the development of the project. Thanks are also expressed to Italian Society of Homeopathy and Integrated Medicine (SIOMI) for scientific support.

\section{References}

1. Bernardini S, Cracolici F, Ferreri R, Rinaldi M, Pulcri R (2015) Integration between orthodox medicine, homeopathy and acupuncture for inpatients: Three years' experience in the first hospital for Integrated Medicine in Italy. J Tradit Complement Med 5:234-240.

2. Fang J, Chen L, Chen L, Wang C, Keeler CL, et al. (2014) Integrative medicine for subacute stroke rehabilitation: A study protocol for a multicentre, randomised, controlled trial. BMJ Open Dec 4:4.

3. Wade DT, Collin C (1987) The Barthel activities of daily living index: A standard measure of physical disability? Int Dis Studies 10:64-67.

4. Franchignoni FP, Tesio L, Ricupero C, Martino MT (1997) Trunk control test as an early predictor of stroke rehabilitation outcome. Stroke 28: 1382-1385.

5. Imbens GW, Rubin DB (2015) Causal inference in statistics, social, and biomedical sciences. Cambridge University Press, UK.
6. Holland PW (1986) Statistics and causal inference. J Am stat Assoc 81: 945-960.

7. Rosenbaum PR, Rubin DB (1983) The central role of the propensity score in observational studies for causal effects. Biometrika 70: 41-55.

8. Fisher RA (1925) Statistical methods for research workers. Genesis Publishing Pvt Ltd.

9. R Core Team (2015) R: A language and environment for statistical computing. R Foundation for Statistical Computing, Vienna, Austria.

10. Ho DE, King G, Imai K, Stuart EA (2011) Match it: Nonparametric preprocessing for parametric causal inference. J Stat Softw 42: 1-28.

11. Mak JC, Mak LY, Shen Q, Faux S (2009) Perceptions and attitudes of rehabilitation medicine physicians on complementary and alternative medicine in Australia. Intern Med J 39:164-169.

12. Skovgaard L, la Cour S, Kristensen M (2015) Use of complementary and alternative medicine at Danish rehabilitation institutions and drug centres. Ugeskr Laeger 174: 39-41.

13. Pannek J, Pannek-Rademacher S, Wöllner J (2015) Use of complementary and alternative medicine in persons with spinal cord injury in Switzerland: A survey study. Spinal Cord 53: 569-572.

14. Chapman EH, Weintraub RJ, Milburn MA, Pirozzi T, Woo E (1999) Homeopathic treatment of mild traumatic brain injury: A randomized, double-blind, placebo-controlled clinical trial. J Head Trauma Rehabil 14: 521-542.

15. Bell IR (2007) Adjunctive care with nutritional, herbal, and homeopathic complementary and alternative medicine modalities in stroke treatment and rehabilitation. Top Stroke Rehabil 14: 30-39.

16. Sajedi F, Alizad V, Alaeddini F, Fatemi R, Mazaherinezhad A (2008) The effect of adding homeopathic treatment to rehabilitation on muscle tone of children with spastic cerebral palsy. Complement Ther Clin Pract 14: 33-37.

17. Yang A, Wu HM, Tang JL, Xu L, Yang M, et al. (2016) Acupuncture for stroke rehabilitation. Cochrane Database Syst Rev 26: CD004131

18. Li L, Zhang H, Meng SQ, Qian HZ (2014) An updated meta-analysis of the efficacy and safety of acupuncture treatment for cerebral infarction. PLoS One 9: e114057.

19. Alexander DN, Cen S, Sullivan KJ, Bhavnani G, Ma X, et al. (2004) Effects of acupuncture treatment on post stroke motor recovery and physical function: A pilot study. Neurorehabil Neural Repair 18: 259-267.

20. Magnusson G, Ballegaard S, Karpatschof B, Nyboe J (2010) Long-term effects of integrated rehabilitation in patients with stroke: A nonrandomized comparative feasibility study. J Altern Complement Med 16: 369-374.

21. JG Lin, WL Chen (2008) Acupuncture analgesia: A review of its mechanisms of actions. Am J Chin Med 36: 635-645.

22. Chang H, Kwon YD, Yoon SS (2011) Use of acupuncture therapy as a supplement to conventional medical treatments for acute ischaemic stroke patients in an academic medical centre in Korea. Complement Ther Med 19: 256-263.

23. Wang C, Wu Z, Li N, Zhao Y, Tian F, et al. (2014) Clinical curative effect of electric acupuncture on acute cerebral infarction: A randomized controlled multicenter trial. J Tradit Chin Med 34: 635-640.

24. Zhang S, Wu B, Liu M, Li N, Zeng X, et al. (2015) Acupuncture efficacy on ischemic stroke recovery: Multicenter randomized controlled trial in China. Stroke 46: 1301-1306.

25. Usichenko TI, Dinse M, Hermsen M, Witstruck T, Pavlovic D, et al. (2005) Auricular acupuncture for pain relief after total hip arthroplasty A randomized controlled study. Pain 114: 320-327.

26. Wang JF, Bao HX, Cai YH, Zhang JH, Tong PJ (2012) Case-control study on application of auricular acupuncture for the treatment of analgesia during perioperative period in total hip arthroplasty. Zhongguo Gu Shang 25: 220-223.

27. Lan F, Ma YH, Xue JX, Wang TL, Ma DQ (2012) Transcutaneous electrical nerve stimulation on acupoints reduces fentanyl requirement for postoperative pain relief after total hip arthroplasty in elderly patients. Minerva Anestesiol 78: 887-895. 
Citation: Bernardini S, Gottard A, Rinaldi M, Mattei A, Virgili G, et al. (2017) Effect of Integrated Medicine on Physical Performances of Orthopaedic and Stroke Patients: A Propensity Score-Matched Study. Altern Integr Med 6: 255. doi:10.4172/2327-5162.1000255

Page 6 of 6

28. Mikashima Y, Takagi T, Tomatsu T, Horikoshi M, Ikari K, et al. (2012) Efficacy of acupuncture during post-acute phase of rehabilitation after total knee arthroplasty. J Tradit Chin Med 32: 545-548.

29. Crespin DJ, Griffin KH, Johnson JR, Miller C, Finch MD, et al. (2015) Acupuncture provides short-term pain relief for patients in a total joint replacement program. Pain Med 16: 1195-203.

30. Chen CC, Yang CC, Hu CC, Shih HN, Chang YH, et al. (2015) Acupuncture for pain relief after total knee arthroplasty: A randomized controlled trial. Reg Anesth Pain Med. 40: 31-36.

31. Chung-Yuh T, Shih-Liang C, Chih-Cheng W, Chu-Ling C, Wen-Gii C, et al. (2015) Single-blinded, randomised preliminary study evaluating the effects of $2 \mathrm{~Hz}$ electroacupuncture for postoperative pain in patients with total knee arthroplasty. Acupunct Med 33:284-288.

32. Kwang-Ho H, Hyun-Woo C, Eui-Hyoung H, Ki-Bong K, Jin-Hong C, et al. (2013) The use of east-west integrative medicine in a national university hospital setting in Korea: A review of a new routine integrated hospital dataset. Eur J Integr Med 5: 501-505.

33. Brinkhaus B, Wilkens JM, Lüdtke R, Hunger J, Witt CM, et al. (2006) Homeopathic arnica therapy in patients receiving knee surgery: Results of three randomised double-blind trials. Complement Ther Med 4: 237-246. 